\title{
Can cochlear implantation improve neurocognition in the aging population?
}

This article was published in the following Dove Press journal: Clinical Interventions in Aging

\section{Christiane Völter ${ }^{1}$ \\ Lisa Götze' \\ Stefan Dazert ${ }^{1}$ \\ Michael Falkenstein ${ }^{2,3}$ \\ Jan Peter Thomas'}

'Department of Otorhinolaryngology, Head and Neck Surgery, Ruhr

University Bochum, St. ElisabethHospital, Bochum, Germany; '2Institute for Work, Learning and Ageing (ALA), Bochum, Germany; ${ }^{3}$ Leibniz Research Centre for Working Environment and Human Factors, Dortmund, Germany

Correspondence: Christiane Völter Department of Otorhinolaryngology, Head and Neck Surgery, Ruhr University Bochum, St Elisabeth-Hospital, Bleichstr. I5, 44787 Bochum, Germany

Tel +492345098390

Fax +49 2345098391

Email christiane.voelter@ruhr-unibochum.de
Introduction: The relationship between cognition and the ability to hear is well known. Due to changes in demographics, the number of people with sensorineural hearing loss and cognitive impairment is increasing. The aim of this study was to identify the impact of hearing rehabilitation via cochlear implantation on cognitive decline among the aging population.

Patients and methods: This prospective study included 60 subjects aged between 50 and 84 years (mean 65.8 years, $\mathrm{SD}=8.9$ ) with a severe to profound bilateral hearing impairment. A computer-based evaluation of short- and long-term memory, processing speed, attention, working memory and inhibition was performed prior to surgery as well as 6 and 12 months after cochlear implantation. Additionally, speech perception at 65 and $80 \mathrm{~dB}$ (Freiburger monosyllabic speech test) as well as disease-related (Nijmegen Cochlear Implant Questionnaire) and general (WHOQOL-OLD) quality of life were assessed.

Results: Six months postimplantation, speech perception, quality of life and also neurocognitive abilities significantly increased. The most remarkable improvement after 6 months was detected in executive functions such as attention $(p<0.001)$, inhibition $(p=0.025)$ and working memory (n-back: $p=0.002$; operation span task: $p=0.008)$, followed by delayed recall $(p=0.03)$. In contrast, long-term memory showed a significant change of performance only after 12 months $(p=0.021)$. After 6 months, most cognitive domains remained stable, except working memory assessed by the operation span task, which significantly improved between 6 and 12 months $(p<0.001)$. No correlation was found between cognitive results and duration of deafness, speech perception or quality of life.

Conclusion: Cochlear implantation does not only lead to better speech perception and quality of life, but has also been shown to improve cognitive skills in hearing impaired adults aged 50 years or more. These effects seem to be independent of each other.

Keywords: neurocognition, cochlear implantation, hearing rehabilitation, aging

\section{Introduction}

The aging population is growing fast and by 2050 , it is expected that $>20 \%$ of the general population will be 60 years of age and older. ${ }^{1}$ Therefore, the prevalence of a sensorineural hearing loss rises, as $10 \%-20 \%$ of the $50-60$ year olds as well as 30\% of all above 65 years and $55 \%$ of those at the age of 80 years and above are affected by a disabling hearing impairment. ${ }^{2-4}$ Besides this, the neurocognitive processes also change with increasing age. ${ }^{5,6}$ Whereas crystalline intelligence mostly remains stable, fluid intelligence such as working memory, processing speed and inhibition often decline $\mathrm{e}^{7-11}$ with high interindividual variations. ${ }^{12}$ Also, $10 \%$ of 65 -year-old people report mental disabilities, which triples when reaching the eighth decade of life..$^{13}$ Thereby, financial and caregiver burdens will have a significant impact on society in the future and interventions that could delay disease onset or progression by even a modest amount will become important. ${ }^{14}$ 
Recently, large epidemiological studies with several thousand participants have shown that hearing loss is associated with cognitive decline and even dementia. ${ }^{15-20}$ Mild hearing impairment increases the risk to develop a dementia illness within the next 10 years by 2 -fold and a severe impairment even by 5 -fold in comparison to normal hearing adults as reported by Lin and Albert. ${ }^{21}$ Similar results have recently been published by Davies et al in 2017 on 7,856 citizens over the age of 50 in an English longitudinal study of aging. ${ }^{18}$

However, the extent to which hearing impairment impacts on cognition seems to differ according to different neurocognitive subdomains. ${ }^{11}$ So far, the underlying mechanisms are not reliably known: cognitive overload, limited social interaction or common neurobiological causes have previously been studied.22-24

The question of whether hearing rehabilitation by hearing aids or cochlear implants (CIs) can improve neurocognitive performance, especially in the aging adults, is still a matter of research. Despite incoherent findings, several studies support the benefit of hearing aids on cognition, especially with regard to working memory. ${ }^{25,26}$ Usually studies with hearing aids are hampered by the fact that daily use of hearing aid might not be sufficient and cognitive changes might not be detected in the long-term follow-up. ${ }^{25,27-31}$

However, well-designed prospective studies of the impact of cochlear implantation on cognitive abilities are still scarce. ${ }^{32}$ In 2015, Mosnier et al first applied a broad cognitive assessment battery in CI recipients aged between 65 and 85 years in a multicenter study. Episodic memory as well as visuospatial abilities, attention and processing speed as well as mental flexibility were assessed 6 and 12 months after implantation. ${ }^{33}$ In 2016, Cosetti et al reported on significant postoperative benefits in seven CI recipients, which were most evident in verbal and memory domains. ${ }^{34}$ However, tests to encounter working memory were not explored in these studies in detail. Recently, Jayakody et al reported better results with spatial working memory tasks across cochlear-implanted and nonimplanted subjects with severe to profound bilateral hearing impairment. ${ }^{35}$ However, Sonnet et al did not detect an improvement in global cognition by the Mini-Mental State Examination in 16 persons, whereas there was an amelioration in the executive functions evaluated by the Instrumental Activities of Daily Living. ${ }^{36}$ More recently, a longitudinal cohort study on cognitive functioning of $25 \mathrm{CI}$ candidates in the short- and long-term follow-up has been proposed by Claes et al. ${ }^{37}$

The aim of this investigation is to explore the influence of cochlear implantation on neurocognition in a prospective survey of hearing impaired adults aged 50 years and more by analyzing a broad spectrum of neurocognitive abilities such as short- and long-term memory, working memory, attention, processing speed as well as inhibition and verbal fluency.

\section{Patients and methods}

\section{Patients}

In this longitudinal prospective study, all consecutive patients aged 50 years or older suffering from severe to profound bilateral hearing loss and scheduled for cochlear implantation (speech reception score in quiet of $\leq 40 \%$ open set monosyllabic words at $65 \mathrm{~dB}$ sound pressure level in best-aided condition) at the Department of Otolaryngology, Head and Neck Surgery of the Ruhr University Bochum, Germany between 2016 and 2017 were enrolled. All patients underwent unilateral cochlear implantation. Three different devices were used (MedEL Innsbruck, Austria as well as Cochlear Ltd., Sydney, Australia and Advanced Bionics, Valencia, CA, USA). Individuals with severe visual impairment, neurologic or psychiatric disorders or with limited knowledge of the German language were excluded.

In total, 60 patients aged between 50 and 84 years with an average of $65.8(\mathrm{SD}=8.9)$ years and a median $(\mathrm{Mdn})$ age of 66.5 years (68\% CI: $54.8-74.2$ years) had been tested prior to cochlear implantation. Twenty-eight individuals (46.7\%) were younger than 65 years, whereas $32(53.3 \%)$ individuals were 65 years and older (Figure 1).

Out of these, 33 subjects ( 15 males and 18 females) aged between 50 and 82 years (mean [M]: 66.18, SD=8.11 years, Mdn: 67.38 years, $68 \%$ CI: 57.3-71.9 years) were reassessed 6 months after implantation. Thirteen subjects (middle-aged) were $<65$ years, and 20 subjects (the elderly) were 65 years or older. Twenty subjects (11 females and 9 males) with an average age of 68.01 (M: 68.01, SD=7.68, Mdn: 69.37, 68\% CI: 60.97-74.14) years underwent a 12-month follow-up assessment.

Audiometric and cognitive testing were performed preoperatively (t1) as well as at 6 months (t2) and 12 months (t3) after cochlear implantation. All eligible patients gave their written informed consent. The ethical institution of the Ruhr University Bochum approved the study (No. 16-5727-BR).

\section{Audiometric evaluation}

All audiometric tests were assessed in a sound booth (DIN EN ISO 8253). Patients routinely underwent an audiometric assessment prior to cochlear implantation. This consisted of pure tone air and bone conduction audiometry on each ear separately in the frequency range from 0.25 to $8 \mathrm{kHz}$. According to the World Health Organization (WHO) 


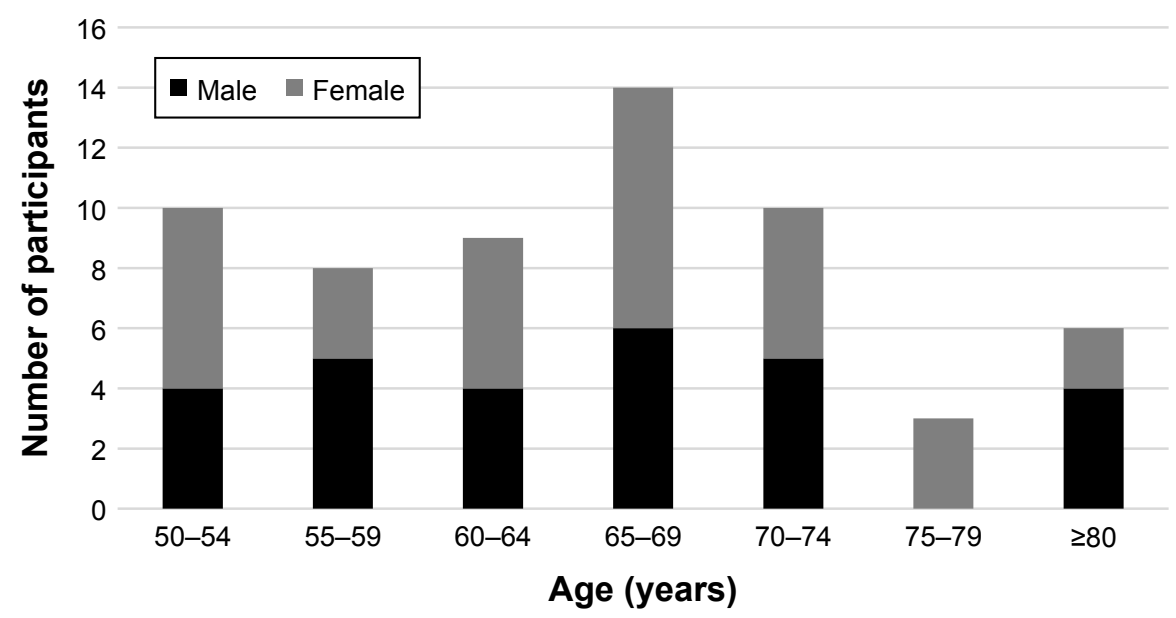

Figure I Demographic data showing the number of participants and gender distribution for each age group in 60 patients who underwent testing prior to cochlear implantation.

definition, severe hearing loss was defined as thresholds of $61-80 \mathrm{~dB}$ and profound hearing loss as $\geq 81 \mathrm{~dB}$ in the following frequencies: $0.5,1,2$ and $4 \mathrm{kHz}$. Additionally, auditory brainstem response audiometry, otoacoustic reflex measures, tympanometry and ipsi- as well as contralateral stapedius reflex measurements between 0.5 and $4 \mathrm{kHz}$ were performed prior to implantation.

Speech comprehension testing was conducted using the German Freiburger monosyllabic speech test in free field at 65 and $80 \mathrm{~dB}$ sound pressure level. This testing was evaluated in unaided and in best-aided conditions using conventional hearing aids before implantation and with the $\mathrm{CI}$ at 6 and 12 months postimplantation.

Additionally, speech perception in noise was measured using the German Oldenburger Sentence Test in an adaptive procedure with fixed background noise of $65 \mathrm{~dB}$ in free field. The signal to noise ratio was adapted in order to understand $50 \%$ of the sentences. Randomly assigned nonsense sentences (subject-verb-number-adjective-object) were applied via free field with speech and noise presented from the front $(\mathrm{S} 0 \mathrm{~N} 0)$.

\section{Neurocognitive testing}

All participants underwent neurocognitive testing using a multimodular computer-based test battery (ALAcog) developed at the Institute of Work, Learning and Ageing in Bochum and the Leibniz Research Centre of Working Environment and Human Factors in Dortmund, Germany. The battery was composed of 10 different subtests based on well-known paper and pencil tests and covered the most important domains of cognition as previously described in detail by Völter et al. ${ }^{38}$

1. M3 test (based on the $\mathrm{d} 2$ test of attention, described by Brickenkamp ${ }^{39}$ ), which predominantly measures attention
2. Recall and delayed recall task (based on the verbal learning and memory test according to Helmstaedter et $\mathrm{al}^{40}$ ) to assess short-term memory function

3. n-back test, which comprises the 0-back and the 2-back task and is used to assess working memory as previously described by Kirchner ${ }^{41}$ and Wild-Wall et $\mathrm{al}^{42}$

4. Operation span task (OSPAN), which also assesses working memory abilities based on the OSPAN ${ }^{43}$

5. Trail A and B tasks, testing processing speed and executive functions (based on Reitan ${ }^{44}$ )

6. Flanker test as described by Eriksen and Eriksen ${ }^{45}$ as well as Wild-Wall et a ${ }^{10}$ to assume inhibitory abilities for compatible and incompatible stimuli

7. Verbal fluency task for evaluation of long-term memory, based on Chicago Word Fluency Test by Thurstone ${ }^{46}$

All instructions were presented visually and patients completed a pre-session prior to the main test procedure. The response time and the number of correct answers were recorded and used to calculate the "inverse efficiency" (IE) performance index. Poorer performance was indicated by higher values. ${ }^{10}$

Premorbid crystallized intelligence was assessed using a multiple choice vocabulary intelligence test (MehrfachwahlWortschatz-Intelligenztest) ${ }^{47}$ with scores between 21 and 30 corresponding to normal intelligence (IQ score between 91 and 109). Additionally, the Dementia Detection test was applied as a brief screening test to rule out patients with a dementia illness, whereby a score of 13-18 points corresponds to normal cognitive abilities, $9-12$ to a slight cognitive decline and $<8$ points to the suspicion of dementia. ${ }^{48}$

\section{Quality of life questionnaires}

After cognitive testing, hearing impaired subjects were invited to answer two different questionnaires evaluating 
their quality of life. The Nijmegen Cochlear Implant Questionnaire (NCIQ) was applied as a measurement of health-related quality of life, which has been proved to be a reliable and valid tool to monitor clinical changes in hearing impaired individuals. ${ }^{49}$ Each of the subjects was asked to provide an answer to 55 statements with scales ranging from 0 (never) to 100 (always) and to 5 statements with scales ranging from 0 (not at all) to 100 (very good) regarding their hearing abilities. The analysis comprises three domains (physical, psychological, social), which can be further distinguished into six subdomains (physical: basic and advanced sound perception, speech production; psychological: self-esteem; social: activity limitations, social interactions). A higher mean score indicates a better health-related quality of life.

Additionally, general quality of life was assessed using a modified scale version of the validated WHOQOL-OLD questionnaire, ${ }^{50,51}$ originally developed as an add-on module for the WHOQOL-BREF ${ }^{52}$ that was adapted for elderly people and their special needs. Across 20 items, patients respond to questions about their sensory abilities, autonomy, past, present and future activities, social participation and about intimacy. For each answer, the patient can select points between 1 and 5, which can either provide a sub- or a total score.

\section{Statistical analysis}

Mean data with standard deviation (SD), median (Mdn) and $68 \%$ confidence interval $(68 \% \mathrm{CI})$ were used to present the results for each patient. Wilcoxon tests were used to compare the pre-evaluation and the 6-months follow-up data for each subtest and sub-measurement of the computer-based test battery as well as for data obtained from each of the questionnaires.

For patients who took part in the 12-months follow-up, repeated analysis of variance measurements was calculated for each subtest and questionnaire with Bonferroni-corrected post hoc analysis to detect the differences between each evaluation.

Furthermore, correlation analyses were done to evaluate the relationship between cognitive performance and hearing ability using Spearman's rho.

All results were interpreted at a significance level of $p<0.05$. Data analysis was performed using Medas (C. Grund, Margetshoechheim, Germany) and SPSS statistics 24 (IBM Corporation). Sample size estimation for different neurocognitive subdomains was completed using Instat Graphpad software $\mathrm{U} 2.0(\alpha=0.05, \beta=0.20)$.

\section{Results \\ Audiometric evaluation}

Pure tone audiogram

4-PTA on the better hearing ear was $80.8 \mathrm{~dB}(\mathrm{SD}=20.3 \mathrm{~dB})$ and on the worse ear $101.7 \mathrm{~dB}(\mathrm{SD}=9.2 \mathrm{~dB})$ preoperatively.

\section{Speech comprehension}

The average speech perception measured by using the German Freiburger monosyllabic test was 3.5\% ( $\mathrm{SD}=6.1$ ) at $65 \mathrm{~dB}$ and $7.8 \%(\mathrm{SD}=13)$ at $80 \mathrm{~dB}$ prior to implantation. Six months after implantation, the average results for monosyllabic words were $50 \%(\mathrm{SD}=20.3)$ at $65 \mathrm{~dB}$ and $65 \%$ $(\mathrm{SD}=18.5)$ at $80 \mathrm{~dB}$ in quiet condition $(p<0.001)$. After 12 months, the speech perception performance rose to $65 \%$ $(\mathrm{SD}=23.7)$ at $65 \mathrm{~dB}$ and $75 \%(\mathrm{SD}=19.6)$ at $80 \mathrm{~dB}$.

The mean speech comprehension in noise $(n=19)$ as measured using the German Oldenburger Sentence Test at 12 months postimplantation was $+0.19 \mathrm{~dB}(\mathrm{SD}=2.08)$ for the best-aided bimodal condition (CI on one side and hearing aid on the other side) and $+2.4 \mathrm{~dB}(\mathrm{SD}=2.1)$ for the $\mathrm{CI}$ alone.

\section{Hearing status}

The duration of hearing loss (time between the first hearing aid use and cochlear implantation) was on average 25.4 years (range: $1-55$ years). Thirty of the 33 subjects were treated bimodally (CI on one side, hearing aid on the contralateral side), 1 patient was cochlear-implanted bilaterally and 2 were unilaterally cochlear-implanted without hearing rehabilitation on the contralateral side. Twenty-eight patients received a MedEL implant system (Sonata/Synchrony, Flex28/ Standard), four subjects received an Advanced Bionics device (High Res 90K, Advantage) and in one patient, a Cochlear Ltd. device (CI512, Contour Advance) had been delivered.

\section{Sample size estimation}

The number of patients necessary to estimate a significant difference $(p<0.05)$ between pre- and postoperative values was calculated by power analysis $(\alpha=0.05, \beta=0.20)$ and varied from 25 subjects for the n-back, 58 with regard to the attention and the delayed recall task as well as 39 subjects for the OSPAN subtest. Some tasks such as the Trail A and B and the immediate recall and inhibitory response to compatible stimuli were found to require a larger number of subjects due to the slight differences between pre- and postoperative performance.

\section{Neurocognitive abilities In general}

Crystallized intelligence as measured by the MehrfachwahlWortschatz-Intelligenztest test showed an IQ score of 101 


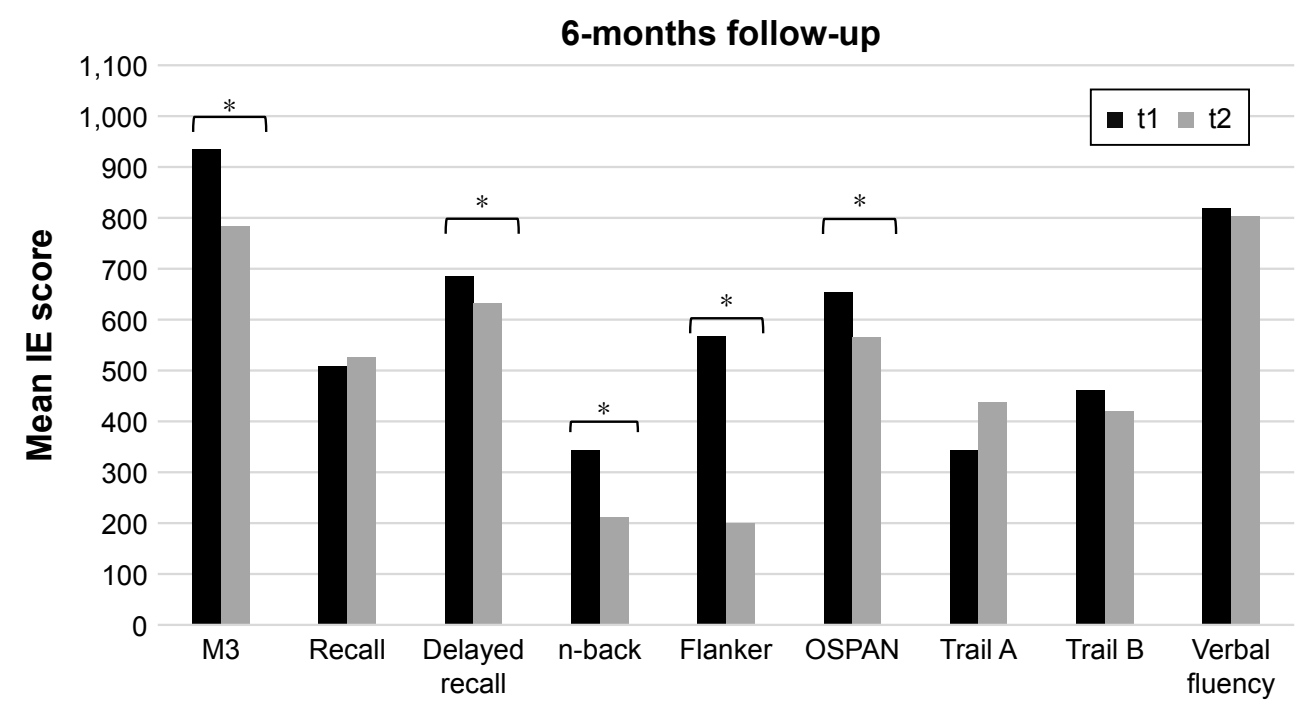

Subtests

Figure 2 Mean performance prior to implantation $(\mathrm{t} l)$ and at 6 months postimplantation $(\mathrm{t} 2)$ for $\mathrm{n}=33$ subjects. Note: Parentheses with $*$ indicate significance of $p<0.05$.

Abbreviations: IE, inverse efficiency; OSPAN, operation span task.

on average (28.3 [SD=3.6] points; range: $21-35$ points). No sign of dementia illness was recognized using the Dementia Detection test with an average score of $17(\mathrm{SD}=1.5)$ points (range: $13-18$ points).

\section{Neurocognitive subtests}

\section{Six-months follow-up}

As shown in Figure 2 changes were observed across the different neurocognitive domains.

The IE performance (Table 1) increased the most with regard to the M3 ( $p=0.00027)$. The reaction times declined from 860.97 to $742.89 \mathrm{~ms}(p=0.0015)$, the number of processed letters in a given time increased from 86.79 to 97.55 $(p<0.001)$ and the number of correct responses increased from 79.61 to $92.67(p<0.001)$.

Table I Mean IE of the subtests for 33 subjects prior to implantation $(\mathrm{tl})$ and at 6 months postimplantation (t2)

\begin{tabular}{llll}
\hline Subtest & IE tI (SD) & IE t2 (SD) & p-value \\
\hline M3 & $935.73(36 I .16)$ & $783.94(350.67)$ & $0.00027^{*}$ \\
Recall & $508.18(229.40)$ & $525.45(179.17)$ & 0.84 \\
Delayed recall & $685.15(207.06)$ & $631.5 I(165.53)$ & $0.03^{*}$ \\
n-back & $342.94(237.33)$ & $212.21(120.03)$ & $0.0022^{*}$ \\
Flanker & $566.31(1,620.05)$ & $199.44(484.66)$ & $0.025^{*}$ \\
OSPAN & $654.37(387.25)$ & $564.75(293.4)$ & $0.0077^{*}$ \\
Trail A & $343.93(190.95)$ & $437.65(177.8)$ & 0.96 \\
Trail B & $462.02(277.00)$ & $419.21(255.03)$ & 0.42 \\
Verbal fluency & $818.182(80.57)$ & $803.79(69.19)$ & 0.15 \\
\hline
\end{tabular}

Note: $p$-value indicates differences between test sessions with significance $(*)$ of $p<0.05$.

Abbreviations: IE, inverse efficiency; OSPAN, operation span task.
The memory functions measured by the immediate and delayed recall tasks did not show a significant difference in the number of correctly remembered words in the immediate recall task between premeasurement and 6-months follow-up $(p=0.84)$. In contrast, there was a significant difference in delayed recall with an average increase from 4.6 (preoperative) to 5.55 (6 months postoperative, $p=0.03$ ).

With regard to the $n$-back performance, positive changes were seen ( $p=0.0022$ ), with the 2-back task between the correctly recognized target letters from $t 1$ to $t 2(p=0.0044)$. There was also a significant decrease in the number of missed trials that could be detected 6 months after cochlear implantation ( $p=0.0052$ ). None of the results from the other measurements of 2-back or 0-back tasks were significant ( $p \geq 0.078$ ).

An improvement of the inhibitory abilities was seen with the Flanker performance $(p=0.025)$. Detailed analyses revealed improved incompatible Flankers between $\mathrm{t} 1$ and $\mathrm{t} 2$ ( $p=0.04$ ), while compatible Flankers did not show any significance ( $p=0.93)$. For reaction times, there were no differences in compatible $(p=0.51)$ and incompatible trials $(p=0.63)$ measured preoperatively and at the 6-months follow-up.

The number of correct responses in compatible trials did not differ across pre- and postoperative measurements $(p=0.17)$. For incompatible arrows, the number of correct responses rose from 26.59 to 29.93 on average over time, indicating a slight but not a significant benefit $(p=0.065)$. Similar results were found for false responses to incompatible arrows $(p=0.059)$. For all other sub-measurements of the Flanker 
task, no significant effects occurred ( $p \geq 0.27$ ). Nevertheless, a decrease of false alarms (eg, pressing button at the wrong time) was observed in the number of false responses on average per subject (3.63 versus 2.63, $p=0.45$ ).

As shown in Table 1, the results from the OSPAN significantly increased 6 months after implantation $(p=0.0077)$. In terms of the mean number of correct symbols per trial, a significant benefit was observed between the preoperative and 6 months postoperative measurements (1.86 versus $2.86, p<0.001)$. The mean reaction time for calculations decreased over time ( $p=0.65$ ). However, the number of correctly calculated tasks decreased and the number of false calculated tasks increased at the same time ( $p=0.0063$ and $p=0.0076$, respectively).

The mean IE for Trail A did not increase significantly ( $p=0.96$ ). The number of patients who were able to finish the whole test within the time frame increased from 26 patients at $\mathrm{t} 1$ to 27 at $\mathrm{t} 2$. More number of subjects finished at least half of the test postoperatively compared to preoperatively (32 versus 27 subjects, respectively). Fourteen subjects in Trail B completed all labels at the 6-months follow-up, compared to 13 prior to implantation. The number of participants who responded to at least half of the labels remained unchanged (26 subjects). The IE decreased slightly, but not significantly $(p=0.42)$. The difference in IE between Trail B and A slightly increased from 181.54 to $194.0(p=0.51)$.

Concerning verbal fluency, the number of correctly named words 6 months after the cochlear implantation increased on average from 6.06 to $6.67(p=0.15)$.

\section{Twelve-months follow-up}

Most subtests remained stable between 6 and 12 months postimplantation, as shown in Figure 3.
M3 performance (IE) significantly changed between $\mathrm{t} 1$ and $\mathrm{t} 2$, but only slightly between $\mathrm{t} 2$ und $\mathrm{t} 3$ ( $p=0.005$ and $p=1$, respectively). Raw data revealed more correctly selected items for the M3 (t1: 75.85; t2: 89.75; t3: 91.8) between $\mathrm{t} 1$ and $\mathrm{t} 2(p<0.001)$ and no further improvement at 1 year postimplantation $(p=0.1)$.

Memory tasks showed biased results. The number of immediately recalled words did not significantly increase between $\mathrm{t} 1$ and $\mathrm{t} 2(p=0.67)$ and between $\mathrm{t} 2$ and $\mathrm{t} 3(p=1 ; \mathrm{t} 1$ : 6.15 words, t2: 6.6 words, t3: 6.75 words), whereas in delayed recall, 3.85 words on average were remembered at the first appointment and 5.55 words at $\mathrm{t} 2(p=0.003)$. No further improvement was detected at 12 months postimplantation (5.4 average words, $p=1$ ).

Patients were able to achieve a significantly higher number of correct responses on average for the 2-back task at 6 months after hearing rehabilitation ( $\mathrm{t} 1: 23.95$ letters, $\mathrm{t} 2$ : 28.32 letters; $p=0.001$ ). After 12 months, the performance was found to be stable with 27.53 letters correctly recognized ( $p=0.76)$.

In contrast, the OSPAN performance (IE) significantly improved between 6 and 12 months postoperatively ( $\mathrm{t} 1$ : 629.68, t2: 567.68, t3: 499.95; $p=0.046)$. Raw data showed that the mean number of correctly calculated tasks increased between 6 and 12 months for this subgroup of 20 patients (t2: 37.84 tasks, $\mathrm{t} 3: 39.1$ tasks; $p=0.12$ ). A significant increase in the number of correctly remembered letters or numbers per trial on average was detected between $t 1$ and $t 2$ and between $\mathrm{t} 1$ and $\mathrm{t} 3$ ( $\mathrm{t} 1: 1.79$ letters/numbers, $\mathrm{t} 2: 2.80$ letters/numbers, t3: 2.93 letters/numbers; each $p<0.001$ ).

The Flanker performance (IE) significantly ameliorated between baseline and 12 months assessment (t1: 411.72, t2: 178.61, t3: 142.5; $p=0.008)$. Detailed analysis revealed that

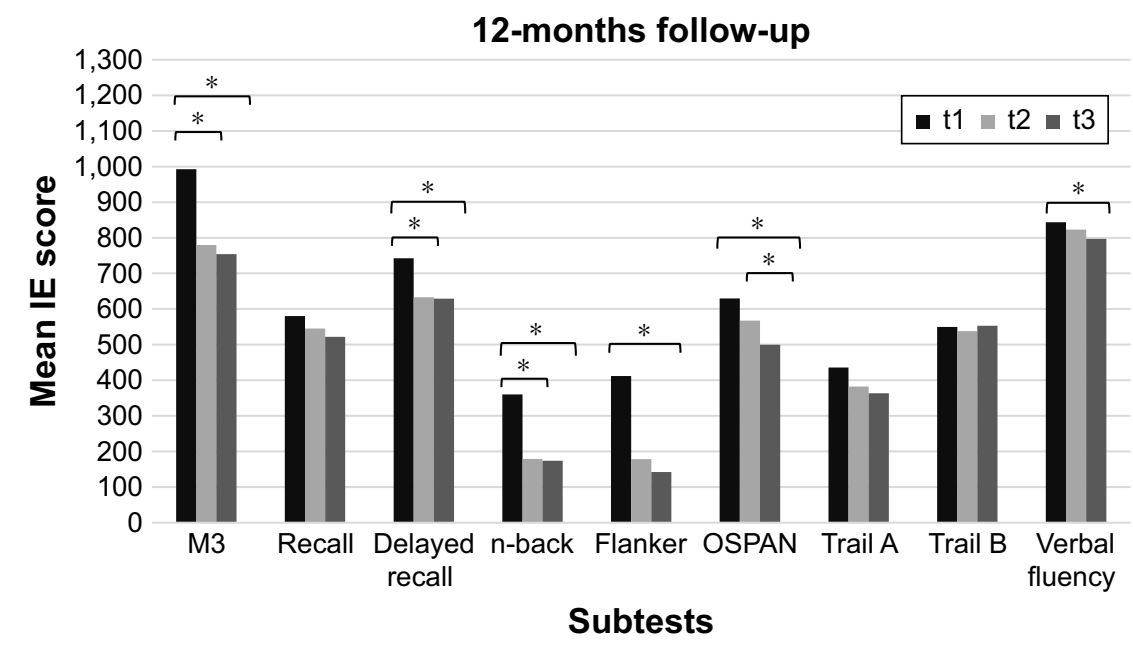

Figure 3 Mean performance IE prior to implantation ( $\mathrm{tl}$ ) and at 6 months ( $\mathrm{t} 2$ ) and 12 months ( $\mathrm{t} 3$ ) postimplantation for $\mathrm{n}=20$ subjects. Note: Parentheses with * indicate significance of $p<0.05$.

Abbreviations: IE, inverse efficiency; OSPAN, operation span task. 
incompatible Flanker slightly ameliorated between $\mathrm{t} 1$ and $\mathrm{t} 2$ ( $p=0.23$ ) and significantly between $\mathrm{t} 1$ and $\mathrm{t} 3(p=0.04)$, whereas compatible Flanker did not change ( $\mathrm{t} 1$ versus $\mathrm{t} 3 p=0.55$ ).

Trail A and B tasks did not show any significant improvement over the three measurements ( $p=0.056$ and $p=0.94$, respectively).

Additionally, verbal fluency performance (IE) revealed a significant benefit not earlier than after 12 months ( $p=0.021)$. Also, 5.52 nouns were named on average during the baseline assessment test. This number slightly increased to 6.05 words after 6 months and reached significance after 12 months, with the subjects naming 6.9 nouns correctly $(p=0.009)$.

\section{General quality of life}

General quality of life as obtained using the WHOQOLOLD significantly improved at 6 months after cochlear implantation ( $\mathrm{t} 1: 72.52$ [SD=7.37], t2: $75.41[\mathrm{SD}=7.56]$; $p=0.027)$. This was also true for the sensory functions (t1: $9.9[\mathrm{SD}=2.91], \mathrm{t} 2: 13.03[\mathrm{SD}=2.89] ; p<0.001)$ and the autonomy-related questions ( $\mathrm{t} 1: 15.1$ [SD=2.83], $\mathrm{t} 2$ : 15.97 [ $\mathrm{SD}=1.72] ; p=0.026)$. In contrast, the level of activities $(p=0.60)$, social participation $(p=0.81)$ and intimacy $(p=0.16)$ did not change significantly.

At 12 months postimplantation, no further improvements were observed with the overall score $(p=1.0)$, sensory abilities $(p=0.31)$, autonomy $(p=1.0)$, activities $(p=1.0)$, social interaction $(p=0.92)$ or intimacy $(p=1.0)$.

\section{Health-related quality of life}

Health-related quality of life as measured using the NCIQ significantly improved across all domains at 6 months after implantation (Figure 4). Social and physical aspects increased by $17.7 \%(p<0.001)$ and $17.22 \%(p<0.001)$, respectively, between $\mathrm{t} 1$ and $\mathrm{t} 2$. The psychological quality of life increased by $13.3 \%(p<0.001)$ between $\mathrm{t} 1$ and $\mathrm{t} 2$.

On analyzing the different subdomains, quality of life was found to change significantly. Basic and advanced sound perception abilities improved by $20.3 \%$ and $20.4 \%$, respectively, activity limitation, social interaction and self-esteem improved by $17.64 \%, 17.83 \%$ and $13.3 \%$, respectively $(p<0.001)$. Speech production, based on the highest preoperatively score, increased ( $\mathrm{t} 1: 66.02[\mathrm{SD}=17.36], \mathrm{t} 2: 76.8$ [SD=15.32]; $p=0.001)$ too.

At 12 months postimplantation, the quality of life scores remained consistent across all subtests (physical, social and psychological) and no further increase was observed $(p=0.88$, $p=1.0$ and $p=0.092$, respectively).

\section{Individual improvement}

Regarding continuous age and the level of improvement without taking the baseline performance into account, elderly subjects showed similar increases as those observed in younger individuals in all subtests investigated. In the 2-back, older subjects even had a significantly greater benefit than that observed in younger subjects $(r=-0.37, p=0.035)$ using continuous age regression analysis.

The level of individual benefit between the different test sessions was significantly better for subjects with poor baseline results. This was particularly evident in the recall tasks (immediate recall: $r=-0.7$, delayed recall: $r=-0.58$ ), the attention $(r=-0.69)$, the 2-back $(r=-0.6)$ and the Trail

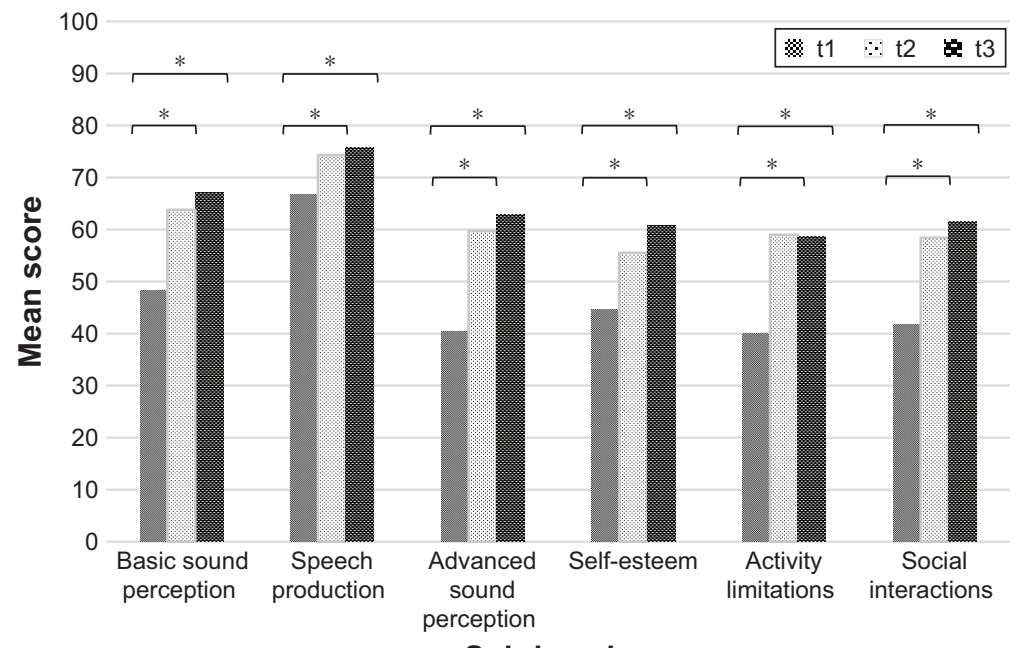

Subdomains

Figure 4 Mean scores for the Nijmegen Cochlear Implant Questionnaire prior to implantation ( $\mathrm{t} I)$ and at 6 months (t2) and I2 months (t3) postimplantation for $\mathrm{n}=20$ subjects. Note: Parentheses with * indicate significance of $p<0.05$. 
A $(r=-0.7)$ and B $(r=-0.56)$ tests, with $p<0.001$ for each subtest. Verbal fluency $(r=-0.47, p=0.0061)$ and incompatible Flankers also improved more in subjects with a low preoperative performance in comparison to those with a high preoperative performance $(r=-0.48, p=0.0058)$. The OSPAN also $(r=-0.34, p=0.054)$ correlated with baseline performance, although not being statistically significant.

Looking only at subjects with poor baseline performance, the benefit of a CI was higher in subjects above the age of 65 years than in the middle-aged group (Table 2). There was a significant improvement with regard to the baseline results for the recall $(r=-0.78)$ and the delayed recall $(r=-0.66)$ and the Flanker test $(r=-0.78)$ in the older group. The M3 and the n-back improved in both age groups significantly. Verbal fluency was the only test that improved more in middleaged people with poor baseline results than in elderly adults $(r=-0.82, p<0.001)$.

\section{Correlation between hearing abilities and cognition measures}

\section{Six-months follow-up}

No significant correlation was found in preoperative IE performance between cognition and speech perception in quiet or in noise. The reaction time of compatible Flankers after 6 months significantly correlated with the duration of hearing loss $(r=0.359, p=0.044)$.

\section{Twelve-months follow-up}

Even after 12 months, we could not detect any significant correlation between cognitive performance and audiologic measurements regarding memory tasks, verbal fluency, working memory, M3 or Trail tasks. However, subjects with good

Table 2 Improvements observed across subtests from the baseline results in terms of age

\begin{tabular}{|c|c|c|c|c|}
\hline \multirow[t]{2}{*}{ Subtest } & \multicolumn{2}{|c|}{$<65$ years old } & \multicolumn{2}{|c|}{$\geq 65$ years old } \\
\hline & Rho & $p$-value & Rho & $p$-value \\
\hline M3 & -0.83 & $<0.001 *$ & -0.74 & $<0.001 *$ \\
\hline Recall & -0.53 & 0.059 & -0.78 & $<0.001 *$ \\
\hline Delayed recall & -0.38 & 0.2 & -0.66 & $0.0015^{*}$ \\
\hline n-back & -0.60 & $0.029 *$ & -0.70 & $<0.001 *$ \\
\hline Flanker & -0.08 & 0.8 & -0.78 & $<0.00$ I* \\
\hline OSPAN & -0.27 & 0.36 & -0.31 & 0.2 \\
\hline Trail A & -0.17 & 0.58 & -0.34 & 0.26 \\
\hline Trail B & -0.26 & 0.39 & 0.10 & 0.73 \\
\hline Verbal fluency & -0.82 & $<0.001 *$ & -0.30 & 0.31 \\
\hline
\end{tabular}

Note: ${ }^{*}<<0.05$.

Abbreviation: OSPAN, operation span task. compatible Flanker performance prior to implantation were able to achieve better speech perception scores at $65 \mathrm{~dB}$ after 12 months $(r=-0.49, p=0.039)$.

\section{Correlations between quality of life and hearing abilities}

No correlation was found between the Nijmegen hearing specific questions concerning basic and advanced sound perception and objective hearing data after 6 or 12 months. However, improvements in self-esteem after 1 year were associated with speech performance at $65 \mathrm{~dB}$ after 12 months ( $r=0.048, p=0.046$ ). Furthermore, the advanced sound perception measured preoperatively correlated with speech perception at $80 \mathrm{~dB}$ for $\mathrm{t} 2(r=0.438, p=0.036)$ and sentence recognition in noise at $\mathrm{t} 3(r=-0.646, p=0.004)$. In addition, the duration of hearing loss was also significantly associated with the basic sound perception results at $\mathrm{t} 1(r=-0.349$, $p=0.047)$. The general quality of life did not reveal any correlation with speech perception in quiet or in noise or with the duration of hearing loss.

\section{Discussion}

Hearing rehabilitation has considerably changed over the last few years. Until then, severe to profound hearing loss could not be sufficiently treated. However, the development of CI provides the opportunity to restore hearing abilities even in deaf patients. ${ }^{53-55}$ In the past few years, the link between hearing and cognition was extensively studied and the impact of the top-down mechanisms on speech understanding, especially in adverse listening situations or in hearing impaired individuals, has gained further attention. ${ }^{56-58}$ Whereas working memory does not significantly contribute to normal hearing listeners' sentence recognition score below the age of 40 years, this typically becomes evident at an older age. ${ }^{59}$ In hearing impaired individuals, working memory capacity compensates for this mismatch by overcoming the disruption of the automatic implicit process according to the Ease of Language Understanding model. ${ }^{60}$

On the other hand, neurocognitive functions are also influenced by hearing status. Large epidemiological analyses such as the one reported by Lin et al on 347 individuals aged 55 years and older demonstrated that hearing impaired performed worse in memory, global cognitive and executive functions tests in comparison to normal hearing subjects. ${ }^{17}$ Electroencephalographic studies strengthened these results by showing decreased activation patterns in hearing impaired adults following auditory stimuli in temporal areas, usually responsible for auditory processing, and an increased 
response in cortical frontal areas, which are associated with executive functions. ${ }^{82}$

Due to the growing number of elderly people with hearing loss and cognitive decline, the question arises whether hearing rehabilitation might counteract neurocognitive changes in the aging population.

In the past, a series of investigations was undertaken to analyze the effect of aural rehabilitation on cognitive performance through digital devices. Most of them showed a benefit, but some revealed conflicting results. ${ }^{28-30,36}$ Only a few studies performed so far used a comprehensive test battery. ${ }^{33-35,37}$ The Repeatable Battery for the Assessment of Neuropsychological Status, proposed by Claes et $\mathrm{al}^{37}$ and used by Cosetti et al, ${ }^{34}$ strongly focus on memory and visuospatial abilities. Others such as Mosnier et al have chosen a mixture of different subtests covering different neurocognitive domains such as attention, short-term memory as well as processing speed, verbal fluency and visuospatial properties. ${ }^{33}$ However, working memory, which is important to understand speech in adverse situations, especially in the elderly, as well as inhibitory skills were not analyzed in detail.

Some studies only performed screening tests such as the Mini-Mental State Examination or the Montreal Cognitive Assessment. ${ }^{31,61}$ Although proven to be sensitive enough to screen for dementia illness, these tests might not be suitable to detect slight cognitive changes in aging subjects with otherwise normal cognitive properties. ${ }^{62}$ This might explain why Sonnet et al could not detect any neurocognitive improvement in $16 \mathrm{CI}$ recipients aged between 65 and 80 years at 6 months after implantation. ${ }^{36}$

Based on the results of our study, hearing rehabilitation via cochlear implantation has a strong effect, especially on executive functioning. Executive functions including attention, working memory, inhibition and mental flexibility are a basic part of everyday life. As they underlay an age-related decline, they account for $54 \%$ of variance described in daily activities across the elderly population. ${ }^{10,63-66}$

Attention improved the most with the reaction time significantly decreasing and the average number of correct processed letters increasing from 79.6 to 92.6 after 6 months. Similar findings were reported by Mosnier et al who also found significant changes in reaction time after 6 months and a decrease in errors 12 months after cochlear implantation. ${ }^{33}$

Additionally, the ability to suppress irrelevant information is particularly important for individuals with hearing impairment, in order to filter out lexical information. ${ }^{67}$ Inhibition, as assessed by the Flanker, significantly improved for incompatible stimuli. Raw data revealed that the number of correct answers, but not the reaction time, improved. In contrast, Jayakody et al described a change in reaction time, but not in error rate, between a group of severe hearing impaired subjects and CI recipients at a 12-months follow-up. ${ }^{35}$ This discrepancy might be due to slight differences of the subtests used. Jayakody et al applied a Stroop-based test, which measures the ability to ignore response interferences, but not distracting information as in our study. ${ }^{68}$

Another important executive function is working memory, which accounts for storing and processing information. ${ }^{69}$ Hearing impaired individuals are more dependent on working memory abilities in adverse listening situations than normal hearing individuals. ${ }^{70}$ Providing adequate auditory input might reduce the cognitive load caused by hearing loss. After hearing rehabilitation, the working memory performance significantly improved with the n-back and the OSPAN, which assess different aspects of working capacity. ${ }^{71,72}$ This may be explained by the fact that more cognitive resources are available when cognitive load is reduced. But whereas the n-back only increased after 6 months, the highly demanding OSPAN continued to increase even after 12 months. This was primarily due to a phonological improvement rather than to a shorter reaction time.

The impact of hearing rehabilitation on Trail tasks still remains unclear. Our study revealed only minimal changes of Trail A and B in the follow-up, which might be too small to find statistically significant differences in a reasonable sample size as shown by the sample size estimation. In general, Trail tasks measure numerous overlapping cognitive abilities such as visual scanning, motoric speed and cognitive flexibility. ${ }^{73}$ Whereas Trail $\mathrm{B}$ is a more cognitive demanding task, Trail A is highly motoric dependent. According to a study by Oliveira et al, Trail A subtest clearly revealed a training effect in older individuals. ${ }^{74}$ Therefore, limited motoric skills may have masked a slight benefit in our study. The study by Mosnier et al also included Trail tasks and described an improvement for poor performers in Trail A after 12 months and in Trail B both after 6 and 12 months. ${ }^{33}$ In contrast, Cosetti et al noted similar observations to those in our study and suggested a medical history of breast cancer in some of the subjects to be responsible for the decline. ${ }^{34}$

Additionally, both short- and long-term memory benefit from hearing restoration. Subjects included in this study were able to recall significantly more words measured by delayed recall at 6 months postimplantation, whereas performance of 
immediate recall remained unchanged. It can be hypothesized that immediate recall is not sensitive enough to identify mild cognitive changes. Cosetti et al also reported a moderate improvement in memory domains, which was most evident for recognition tasks. However, it should be noted that Cosetti et al presented the words one by one, whereas in our study, all 10 nouns appeared on the screen at the same time. ${ }^{34}$ No significant differences concerning immediate or delayed recognition or recall tasks were detected by Jayakody et al after 6 and 12 months. ${ }^{35}$

Neurocognitive processes already improved significantly after 6 months and remained stable over time. Only longterm memory performance did not increase earlier than 12 months postimplantation. This might be due to the fact that long-term memory as a part of crystalline intelligence is not subjected to fluctuations in the same way as other cognitive domains. ${ }^{11}$ These results are in line with Cosetti et al, who found a moderate increase of semantic fluency 2 years after cochlear implantation using the semantic animal test. ${ }^{34}$ The verbal fluency task, applied in the current study, combines both phonological and semantic aspects by naming animals with a certain letter. One year after implantation, subjects were able to name on average 7.0 words in contrast to 5.5 at baseline.

Besides, subjects with poor cognitive results at baseline measures improved more than those with better baseline results. This might be due to a ceiling effect with no further increase in subjects presenting with good preoperative performance. However, similar results were also published by Mosnier et al. ${ }^{33}$ Interestingly, subjects aged 65 years or more improved significantly more than the middle-aged group below 65 years. Thus, it might be postulated that especially elderly adults presenting with lower neurocognitive performance will benefit from hearing rehabilitation. However, it is not yet clear to what extent severely cognitive impaired adults might also profit from aural rehabilitation strategies and technical devices. ${ }^{61,75}$

The question of whether cognitive skills might be a predictor of postoperative speech outcome has been discussed in the past with inconsistent results. ${ }^{34,76}$ No correlation between preoperative cognitive abilities and postoperative speech performance in either quiet or in noise conditions was detected in our investigation, which is in contrast to the data shown by Heydebrand et $\mathrm{al}^{77}$ or Moberly et al. ${ }^{78}$

Although hearing restoration is the primary goal, cochlear implantation also has a great influence on the individual's quality of life. Several studies have examined the relationship between improvement in speech understanding and changes in quality of life. Some studies, such as the one by Hirschfelder et al, described a significant association between total NCIQ score, NCIQ advanced sound perception and the Freiburger monosyllabic speech perception test in quiet at $70 \mathrm{~dB}$, as well as between the speech production NCIQ total score and the Hochmaier Schulz sentence test in noise across 56 adult CI users. ${ }^{79}$

However, most studies, such as a recently published metaanalysis covering 13 articles and 715 subjects, revealed no or only a low correlation between speech recognition and quality of life. ${ }^{80}$ Capretta and Moberly, applying three different quality of life measurements to assess a variety of quality of life factors, could not detect any relationship. ${ }^{81}$ Whereas NCIQ is not specifically designed to the specifics of the elderly, the WHOQOL-OLD appears to be a good measurement to explore the quality of life in the aging population. ${ }^{50}$ In our study, postoperative NCIQ scores did not significantly correlate with speech perception at 65 or $80 \mathrm{~dB}$ at 6 months postimplantation. This discrepancy can be explained by the fact that audiologic testing only assesses auditory input, whereas communication in everyday life as measured by quality of life tests relies on a combined audiovisual input via lip reading and hearing as well as on social support.

To sum up, hearing restoration by cochlear implantation not only has an impact on hearing restoration and on the quality of life, but also on neurocognitive abilities, even in subjects with low cognitive performance. Therefore, improved postoperative executive functioning will have a great benefit in daily life, particularly among the elderly population. Whether or not cochlear implantation can reduce the prevalence of dementia illness cannot be answered right now.

\section{Acknowledgments}

We would like to thank to Dr Imme R Haubitz (Würzburg) for statistical support, Ludger Blanke (Leibniz Research Centre for Working Environment and Human Factors, Dortmund and Institute for Work, Learning and Ageing, Bochum) for helpful technical assistance, Dr U Bruene-Cohrs (LWL University Hospital, Department of Psychiatry, Psychotherapy and Preventive Medicine, Ruhr University Bochum) for helpful discussions, Dr Laura Kerr (MedEL) for editing a version of this manuscript, as well as Robert Käppeler (cand. med.) and Milena Pertz (MSc psychol.) from the Department of Otorhinolaryngology, Head and Neck Surgery, Ruhr University Bochum for data collection. 


\section{Disclosure}

CV has received travel expense support from MedEL, SD has received third party funds, payment for lectures and travel expense support from MedEL, and JPT has received travel expense support and payment for lectures from MedEL. The authors report no conflicts of interest in this work.

\section{References}

1. United Nations. World population ageing 1950-2050; 2002 World Assembly on Ageing. Available from: https://www.un.org/esa/population/publications/worldageing19502050/. Accessed December 20, 2017.

2. Roth TN, Hanebuth D, Probst R. Prevalence of age-related hearing loss in Europe: a review. Eur Arch Otorhinolaryngol. 2011;268(8):1101-1107.

3. Gablenz P von, Holube I. Prevalence of hearing impairment in northwestern Germany. Results of an epidemiological study on hearing status (HORSTAT). HNO. 2015;63(3):195-214.

4. World Health Organization Regional Office for South-East Asia. Deafness and Hearing Impairment Survey-Report of the Consultative Meeting of Principal Investigators SEARO, New Delhi, 7-9 May 2001. New Delhi, India: WHO.

5. Yesavage JA, O'Hara R, Kraemer H, et al. Modeling the prevalence and incidence of Alzheimer's disease and mild cognitive impairment. J Psychiatr Res. 2002;36(5):281-286.

6. Ferri CP, Prince M, Brayne C, et al. Global prevalence of dementia: a Delphi consensus study. Lancet. 2005;366(9503):2112-2117.

7. Salthouse TA. Aging and measures of processing speed. Biol Psychol. 2000;54(1):35-54.

8. Verhaeghen P. Aging and executive control: reports of a demise greatly exaggerated. Curr Dir Psychol Sci. 2011;20(3):174-180.

9. Braver TS, Barch DM, Keys BA, et al. Context processing in older adults: evidence for a theory relating cognitive control to neurobiology in healthy aging. J Exp Psychol Gen. 2001;130(4):746-763.

10. Wild-Wall N, Falkenstein M, Hohnsbein J. Flanker interference in young and older participants as reflected in event-related potentials Brain Res. 2008;1211:72-84.

11. Uchida $Y$, Nishita $Y$, Tange C, et al. The longitudinal impact of hearing impairment on cognition differs according to cognitive domain. Front Aging Neurosci. 2016;8:201.

12. Reuter-Lorenz PA, Jonides J, Smith EE, et al. Age differences in the frontal lateralization of verbal and spatial working memory revealed by PET. J Cogn Neurosci. 2000;12(1):174-187.

13. Qiu C, Kivipelto M, von Strauss E. Epidemiology of Alzheimer's disease: occurrence, determinants, and strategies toward intervention. Dialogues Clin Neurosci. 2009;11(2):111-128.

14. Brookmeyer R, Johnson E, Ziegler-Graham K, Arrighi HM. Forecasting the global burden of Alzheimer's disease. Alzheimers Dement. 2007; 3(3):186-191.

15. Heywood R, Gao Q, Nyunt MSZ, et al. Hearing loss and risk of mild cognitive impairment and dementia: findings from the Singapore longitudinal ageing study. Dement Geriatr Cogn Disord. 2017;43(5-6):259-268.

16. Jayakody DMP, Friedland PL, Eikelboom RH, Martins RN, Sohrabi HR. A novel study on association between untreated hearing loss and cognitive functions of older adults: baseline non-verbal cognitive assessment results. Clin Otolaryngol. 2018;43(1):182-191.

17. Lin FR, Ferrucci L, Metter EJ, An Y, Zonderman AB, Resnick SM. Hearing loss and cognition in the baltimore longitudinal study of aging. Neuropsychology. 2011;25(6):763-770.

18. Davies HR, Cadar D, Herbert A, Orrell M, Steptoe A. Hearing impairment and incident dementia: findings from the English longitudinal study of ageing. J Am Geriatr Soc. 2017;65(9):2074-2081.

19. Thomson RS, Auduong P, Miller AT, Gurgel RK. Hearing loss as a risk factor for dementia: a systematic review. Laryngoscope Investig Otolaryngol. 2017;2(2):69-79.
20. Su P, Hsu C-C, Lin H-C, et al. Age-related hearing loss and dementia: a 10-year national population-based study. Eur Arch Otorhinolaryngol. 2017;274(5):2327-2334.

21. Lin FR, Albert M. Hearing loss and dementia-who is listening? Aging Ment Health. 2014;18(6):671-673.

22. Stenfelt S, Ronnberg J. The signal-cognition interface: interactions between degraded auditory signals and cognitive processes. Scand $J$ Psychol. 2009;50(5):385-393.

23. Fortunato S, Forli F, Guglielmi V, et al. A review of new insights on the association between hearing loss and cognitive decline in ageing. Acta Otorhinolaryngol Ital. 2016;36(3):155-166.

24. Fulton SE, Lister JJ, Bush ALH, Edwards JD, Andel R. Mechanisms of the hearing-cognition relationship. Semin Hear. 2015;36(03):140-149.

25. Desjardins JL. Analysis of performance on cognitive est measures before, during, and after 6 months of hearing aid use: a single-subject experimental design. Am J Audiol. 2016;25(2):127-141.

26. Doherty KA, Desjardins JL. The benefit of amplification on auditory working memory function in middle-aged and young-older hearing impaired adults. Front Psychol. 2015;6:721

27. van Hooren SAH, Anteunis LJC, Valentijn SAM, et al. Does cognitive function in older adults with hearing impairment improve by hearing aid use? Int J Audiol. 2009;44(5):265-271.

28. Kalluri S, Humes LE. Hearing technology and cognition. Am J Audiol. 2012;21(2):338-343.

29. Dawes P, Cruickshanks KJ, Fischer ME, Klein BEK, Klein R, NondahlDM. Hearing-aid use and long-term health outcomes: hearing handicap, mental health, social engagement, cognitive function, physical health, and mortality. Int $J$ Audiol. 2015;54(11):838-844.

30. Amieva H, Ouvrard C, Giulioli C, Meillon C, Rullier L, Dartigues J-F. Self-reported hearing loss, hearing aids, and cognitive decline in elderly adults: a 25-year study. J Am Geriatr Soc. 2015;63(10): 2099-2104.

31. Castiglione A, Benatti A, Velardita C, et al. Aging, cognitive decline and hearing loss: effects of auditory rehabilitation and training with hearing aids and cochlear implants on cognitive function and depression among older adults. Audiol Neurootol. 2016;21 (Suppl 1):21-28.

32. Miller G, Miller C, Marrone N, Howe C, Fain M, Jacob A. The impact of cochlear implantation on cognition in older adults: a systematic review of clinical evidence. BMC Geriatr. 2015;15:16.

33. Mosnier I, Bebear J-P, Marx M, et al. Improvement of cognitive function after cochlear implantation in elderly patients. JAMA Otolaryngol Head Neck Surg. 2015;141(5):442-450.

34. Cosetti MK, Pinkston JB, Flores JM, et al. Neurocognitive testing and cochlear implantation: insights into performance in older adults. Clin Interv Aging. 2016;11:603-613.

35. Jayakody DMP, Friedland PL, Nel E, Martins RN, Atlas MD, Sohrabi HR Impact of cochlear implantation on cognitive functions of older adults: pilot test results. Otol Neurotol. 2017;38(8):e289-e295.

36. Sonnet M-H, Montaut-Verient B, Niemier J-Y, Hoen M, Ribeyre L, Parietti-Winkler C. Cognitive abilities and quality of life after cochlear implantation in the elderly. Otol Neurotol. 2017;38(8):e296-e301.

37. Claes AJ, Mertens G, Gilles A, et al. The Repeatable Battery for the Assessment of Neuropsychological Status for Hearing Impaired Individuals (RBANS-H) before and after cochlear implantation: a protocol for a prospective, longitudinal cohort study. Front Neurosci. 2016;10:512.

38. Völter C, Götze L, Falkenstein M, Dazert S, Thomas JP. Application of a computer-based neurocognitive assessment battery in the elderly with and without hearing loss. CIA. 2017;12:1681-1690.

39. Brickenkamp R. Test d2: Aufmerksamkeits-Belastungs-Test. Göttingen, Germany: Hogrefe Verl. für Psychologie; 1962.

40. Helmstaedter C, Lendt M, Lux S. Verbaler Lern- und Merkfähigkeitstest: VLMT; Manual. Göttingen, Germany: Beltz-Test; 2001.

41. Kirchner WK. Age differences in short-term retention of rapidly changing information. J Exp Psychol. 1958;55(4):352-358.

42. Wild-Wall N, Falkenstein M, Gajewski PD. Age-related differences in working memory performance in a 2-back task. Front Psychol. $2011 ; 2: 186$. 
43. Turner ML, Engle RW. Is working memory capacity task dependent? J Mem Lang. 1989;28(2):127-154.

44. Reitan RM. Validity of the trail making test as an indicator of organic brain damage. Percept Mot Skills. 1958;8(3):271-276.

45. Eriksen BA, Eriksen CW. Effects of noise letters upon the identification of a target letter in a nonsearch task. Atten Percept Psychophys. 1974;16(1):143-149.

46. Thurstone LL. Primary mental abilities. Science. 1948;108(2813):585.

47. Lehrl S. MWT-B-Mehrfach-Wortschatz-Intelligenztest. Göttingen, Germany: Hogrefe. 2005.

48. Kalbe E, Kessler J, Calabrese P, et al. DemTect: a new, sensitive cognitive screening test to support the diagnosis of mild cognitive impairment and early dementia. Int J Geriatr Psychiatry. 2004;19(2):136-143.

49. Hinderink JB, Krabbe PF, van den Broek P. Development and application of a health-related quality-of-life instrument for adults with cochlear implants: the Nijmegen cochlear implant questionnaire. Otolaryngol Head Neck Surg. 2000;123(6):756-765.

50. Power M, Quinn K, Schmidt S. Development of the WHOQOL-old module. Qual Life Res. 2005;14(10):2197-2214.

51. Conrad I, Uhle C, Matschinger H, Kilian R, Riedel-Heller SG. Lebensqualitat von alteren Menschen mit leichten kognitiven Storungen. Psychiatr Prax. 2015;42(3):152-157.

52. Whoqol G. Development of the World Health Organization WHOQOLBREF quality of life assessment. Psychol Med. 1998;28(3):551-558.

53. Gaylor JM, Raman G, Chung M, et al. Cochlear implantation in adults: a systematic review and meta-analysis. JAMA Otolaryngol Head Neck Surg. 2013;139(3):265-272.

54. Lin FR, Chien WW, Li L, Niparko JK, Francis HW. Cochlear implantation in older adults. Medicine (Baltimore). 2012;91(5):229-241.

55. Lenarz T, Muller L, Czerniejewska-Wolska H, et al. Patient-related benefits for adults with cochlear implantation: a multicultural longitudinal observational study. Audiol Neurootol. 2017;22(2):61-73.

56. Rudner M, Rönnberg J, Lunner T. Working memory supports listening in noise for persons with hearing impairment. J Am Acad Audiol. 2011;22(3):156-167.

57. Meister H, Schreitmuller S, Grugel L, et al. Cognitive resources related to speech recognition with a competing talker in young and older listeners. Neuroscience. 2013;232:74-82.

58. Rönnberg J, Hygge S, Keidser G, Rudner M. The effect of functional hearing loss and age on long- and short-term visuospatial memory: evidence from the UK biobank resource. Front Aging Neurosci. 2014; $6: 326$.

59. Füllgrabe C, Rosen S. Investigating the role of working memory in speech-in-noise identification for listeners with normal hearing. Adv Exp Med Biol. 2016;894:29-36.

60. Rönnberg J, Lunner T, Zekveld A, et al. The Ease of Language Understanding (ELU) model: theoretical, empirical, and clinical advances. Front Syst Neurosci. 2013;7:31.

61. Ambert-Dahan E, Routier S, Marot L, et al. Cognitive evaluation of cochlear implanted adults using CODEX and MoCA screening tests. Otol Neurotol. 2017;38(8):e282-e284.

62. Mukari SZ-MS, Ishak WS, Maamor N, Wan Hashim WF. A preliminary study investigating the association between hearing acuity and a screening cognitive tool. Ann Otol Rhinol Laryngol. 2017;126(10): $697-705$.
63. Bell-McGinty S, Podell K, Franzen M, Baird AD, Williams MJ. Standard measures of executive function in predicting instrumental activities of daily living in older adults. Int J Geriatr Psychiatry. 2002; 17(9):828-834.

64. Diamond A. Executive functions. Annu Rev Psychol. 2013;64:135-168.

65. Dobbs AR, Rule BG. Adult age differences in working memory. Psychol Aging. 1989;4(4):500-503.

66. Chan RCK, Shum D, Toulopoulou T, Chen EYH. Assessment of executive functions: review of instruments and identification of critical issues. Arch Clin Neuropsychol. 2008;23(2):201-216.

67. Knight S, Heinrich A. Different measures of auditory and visual stroop interference and their relationship to speech intelligibility in noise. Front Psychol. 2017;8:230.

68. Rey-Mermet A, Gade M. Inhibition in aging: what is preserved? What declines? A meta-analysis. Psychon Bull Rev. Epub 2017 Oct 10.

69. Baddeley AD, Hitch G. Working memory. Psychol Learn Motiv. 1974;8: 47-89.

70. Füllgrabe C, Rosen S. On the (Un)importance of working memory in speech-in-noise processing for listeners with normal hearing thresholds. Front Psychol. 2016;7:1268.

71. Redick TS, Lindsey DRB. Complex span and n-back measures of working memory: a meta-analysis. Psychon Bull Rev. 2013;20(6):1102-1113.

72. Oberauer K, Süß H-M, Wilhelm O, Wittman WW. The multiple faces of working memory: storage, processing, supervision, and coordination. Intelligence. 2003;31(2):167-193.

73. Salthouse TA. What cognitive abilities are involved in trail-making performance? Intelligence. 2011;39(4):222-232.

74. Oliveira RSD, Trezza BM, Busse AL, Jacob Filho W. Learning effect of computerized cognitive tests in older adults. Einstein (São Paulo). 2014;12(2):149-153.

75. Lodeiro-Fernández L, Lorenzo-López L, Maseda A, Núñez-Naveira L, Rodríguez-Villamil JL, Millán-Calenti JC. The impact of hearing loss on language performance in older adults with different stages of cognitive function. CIA. 2015;10:695-702.

76. Moberly AC, Bates C, Harris MS, Pisoni DB. The enigma of poor performance by adults with cochlear implants. Otol Neurotol. 2016;37(10): $1522-1528$.

77. Heydebrand G, Hale S, Potts L, Gotter B, Skinner M. Cognitive predictors of improvements in adults' spoken word recognition six months after cochlear implant activation. Audiol Neurootol. 2007;12(4): 254-264.

78. Moberly AC, Houston DM, Harris MS, Adunka OF, Castellanos I. Verbal working memory and inhibition-concentration in adults with cochlear implants. Laryngoscope Investig Otolaryngol. 2017;2(5):254-261.

79. Hirschfelder A, Grabel S, Olze H. The impact of cochlear implantation on quality of life: the role of audiologic performance and variables. Otolaryngol Head Neck Surg. 2008;138(3):357-362.

80. McRackan TR, Bauschard M, Hatch JL, et al. Meta-analysis of qualityof-life improvement after cochlear implantation and associations with speech recognition abilities. Laryngoscope. 2018;128(4):982-990.

81. Capretta NR, Moberly AC. Does quality of life depend on speech recognition performance for adult cochlear implant users? Laryngoscope. 2016;126(3):699-706.

82. Campbell J, Sharma A. Compensatory changes in cortical resource allocation in adults with hearing loss. Front Syst Neurosci. 2013;7:71.
Clinical Interventions in Aging

\section{Publish your work in this journal}

Clinical Interventions in Aging is an international, peer-reviewed journal focusing on evidence-based reports on the value or lack thereof of treatments intended to prevent or delay the onset of maladaptive correlates of aging in human beings. This journal is indexed on PubMed Central, MedLine,
Dovepress

CAS, Scopus and the Elsevier Bibliographic databases. The manuscript management system is completely online and includes a very quick and fair peer-review system, which is all easy to use. Visit http://www.dovepress. com/testimonials.php to read real quotes from published authors. 